\title{
High relative levels of satellite alpha transcripts predict increased risk of bilateral breast cancer and multiple primary cancer in patients with breast cancer and lacking $B R C A$-related clinical features
}

\author{
NAO KAKIZAWA ${ }^{1}$, KOICHI SUZUKI ${ }^{1}$, IKU ABE ${ }^{1}$, YUHEI ENDO ${ }^{1}$, SAWAKO TAMAKI ${ }^{1}$, \\ HIDEKI ISHIKAWA $^{1}$, FUMIAKI WATANABE ${ }^{1}$, KOSUKE ICHIDA ${ }^{1}$, MASAAKI SAITO ${ }^{1}$, \\ KAZUSIGE FUTSUHARA ${ }^{1}$, FUMIO KONISHI ${ }^{2}$ and TOSHIKI RIKIYAMA ${ }^{1}$ \\ ${ }^{1}$ Department of Surgery, Saitama Medical Center, Jichi Medical University, Omiya-ku, Saitama 330-8503; \\ ${ }^{2}$ Nerima-Hikarigaoka Hospital, Nerima-ku, Tokyo 179-0072, Japan
}

Received January 11, 2019; Accepted May 30, 2019

DOI: $10.3892 / o r .2019 .7182$

\begin{abstract}
Patients with breast cancer who undergo surgery have a risk of developing multiple cancers in the contralateral breast and other organs. We previously reported that overexpression of satellite alpha transcripts (SAT) facilitates chromosomal instability, which is involved in the development of multiple tumors in patients with colorectal and gastric cancer. In this study, we elucidated the significance of SAT in the development of multiple tumors in patients with breast cancer. Relative expression of SAT (rSAT) was calculated in normal and tumor tissues from 167 patients. In total, 27 patients developed bilateral breast cancer $(\mathrm{BBC})$ and 27 patients showed multiple primary cancer (MPC), with patients with BBC and MPC showing higher rSAT levels in tumor tissues than those in patients with single breast cancer $(\mathrm{SBC})(\mathrm{P}=0.0312$ and $\mathrm{P}=0.0420$, respectively). Additionally, higher rSAT levels in tumor tissues from patients with $\mathrm{BBC}$ were a significant factor according
\end{abstract}

Correspondence to: Dr Koichi Suzuki, Department of Surgery, Saitama Medical Center, Jichi Medical University, 1-847 Amanuma-cho, Omiya-ku, Saitama-shi, Saitama 330-8503, Japan

E-mail: ksuzbnhm@yahoo.co.jp

Abbreviations: SAT, satellite alpha transcripts; BBC, bilateral breast cancer; SBC, single breast cancer; MPC, multiple primary cancer; HER2, human epidermal growth factor receptor 2; TNBC, triple-negative breast cancer; CPM, contralateral prophylactic mastectomy; BRCA, breast cancer susceptibility gene; rSAT, relative expression of satellite alpha transcripts; RDL, relative demethylation level; ROC, receiver operating characteristic; LINE-1, long interspersed nucleotide element-1; IPMN, intraductal papillary mucinous neoplasm; CI, confidence interval; OR, odds ratio; HR, hazard ratio

Key words: satellite alpha transcripts, bilateral breast cancer, multiple primary cancer, field cancerization to univariate analysis, and multivariate analysis showed that $\mathrm{rSAT}>1.5$ was a significant predictor of MPC [hazard ratio (HR): 2.96; $\mathrm{P}=0.0243$ ); however, we did not clarify the involvement of SAT in normal tissues. Excluding 71 patients with $B R C A$-related clinical features, rSAT levels were higher in patients with BBC and MPC than in patients with SBC in tumor tissues and normal tissues $(\mathrm{P}<0.05)$. Significant predictors according to univariate analysis included $\mathrm{rSAT}>1.5$ in tumor tissues, rSAT $>2.4$ in normal tissues, and $\mathrm{T}<2$, whereas those for multivariate analysis included rSAT $>2.4$ in normal tissues for BBC (HR: 22.7; $\mathrm{P}=0.00120)$ and MPC (HR: 13.0; $\mathrm{P}=0.00601$ ). Our data indicated that patients with breast cancer and high rSAT levels in their breast tissues exhibit a 10- to 20 -fold increased risk for the development of multiple cancers when harboring no BRCA-related clinical features.

\section{Introduction}

Patients with breast cancer who undergo surgery are likely to develop a second cancer in the contralateral breast, with a 2- to 6-fold increased risk of contralateral breast cancer (1-5). Additionally, patients with breast cancer have a generally increased risk of developing multiple primary cancer (MPC) in other organs, such as ovarian, pancreatic and skin cancer (6). For these reasons, patients with breast cancer often consider prophylactic surgery for the contralateral breast and other organs. The registry of contralateral prophylactic mastectomy (CPM) has more than doubled over a 6 -year period $(7,8)$, and the proportion of breast-conserving procedures for the treatment of early stage breast cancer has declined accompanied by a compensatory increase in the number of CPMs (9). Although patients with hereditary breast cancer caused by a germline mutation in the breast cancer susceptibility gene $(B R C A)$ are at high risk of developing multiple tumors, the mechanisms underlying the increased risk of apparently non-hereditary multiple primary breast cancer have not been elucidated.

Multiple cancers can arise simultaneously in regions of normal tissue containing certain genetic and epigenetic 
alterations (10). This phenomenon is exemplified by 'field cancerization' (11), which makes common embryological regions susceptible to neoplasms. Our previous study demonstrated that global DNA demethylation was associated with genomic instability in gastrointestinal cancer (12). Although genetic alterations are found in only a minor fraction of cells from normal tissue $(11,13)$, somatic epigenetic alterations are commonly detected in normal tissues adjacent to various types of cancers (14-20). Our previous study evaluating levels of DNA demethylation in common targets [i.e., repetitive sequences in the whole genome (10), such as long interspersed nucleotide element-1 (LINE-1)] demonstrated that DNA demethylation of LINE-1 is associated with a predisposition to multiple tumors in patients with colorectal cancer (10); however, the general significance of LINE-1 demethylation in carcinogenesis remains unknown. Additionally, we evaluated satellite DNA comprising highly repetitive noncoding sequences located in centromeric regions and implicated in chromosomal stability (21).

Recent studies have provided insight into the role of noncoding DNA in cancer susceptibility $(10,21)$, although these regions are poorly transcribed due to heterochromatin structure. The appropriate transcription of satellite regions is essential for accurate chromosomal segregation (22), and elevated satellite-DNA expression has been observed in various epithelial tumors (23), with the overexpression of satellite RNA inducing abnormal segregation of chromosomes in experimental studies (24). Additionally, we demonstrated that overexpression of satellite alpha transcripts (SAT) leads to chromosomal mis-segregation in normal mammary epithelial cells, thereby enhancing chromosomal instability (25). Moreover, SAT expression levels correlate with DNA hypomethylation levels of SAT in both normal and tumor tissues (25), whereas demethylation of satellite DNA in normal gastric tissues increases susceptibility to multiple gastric cancers (21). These results suggest that excessive satellite RNA plays an important role in carcinogenesis and could be involved in the mechanism underlying the development of multiple tumors as a result of field cancerization.

In the present study, we evaluated the role of SAT in field cancerization and cancer development in the bilateral breast or MPCs in other organs in patients with breast cancer.

\section{Materials and methods}

Patients and specimens. Samples of tumor tissues and normal tissues without cancerous mammary glands were collected from 167 female patients who underwent incisional biopsy or a surgical procedure for breast cancer diagnosis and treatment from July 2015 to July 2017 at Saitama Medical Center, Jichi Medical University (Saitama, Japan). The sample sizes were 165 tumor tissues and 109 normal tissues. The tumor tissue samples were obtained during a surgical operation or preoperative biopsy when patients were candidates for neoadjuvant chemotherapy. When tumor tissue samples were obtained, ultrasonography and 14G biopsy needle (ACECUT; TSK Laboratory, Tochigi, Japan) were used both in preoperative biopsy and surgical operations for accuracy. Normal tissues were also collected from patients with breast cancer during the surgical operation and defined as tissues at least $3 \mathrm{~cm}$ from the tumor and nipple and microscopically identified as normal mammary glands according to histologic examination of hematoxylin and eosin-stained sections. All tissue specimens were immediately soaked in RNAlater (Ambion), and after 24 to $48 \mathrm{~h}$, removed from RNAlater and stored at $-80^{\circ} \mathrm{C}$.

Clinical and pathological findings are presented in Table IA. Family history was defined as positive when one or more relatives of first and/or second degree had a medical history of breast cancer. Human epidermal growth factor receptor 2 (HER2) testing was performed according to the American Society of Clinical Oncology/College of American Pathologists guidelines (26). HER2 status was determined in all patients with invasive breast cancer on the basis of one or more HER 2 test results (negative, equivocal, or positive). HER2-positive status was defined as an area of the tumor with $>10 \%$ contiguous and homogeneous tumor cells. If the results were equivocal, reflex testing was performed using fluorescence in situ hybridization to define HER2-positive or -negative status (26). Triple-negative breast cancer (TNBC) was characterized as cancer exhibiting low expression of estrogen receptor, progesterone receptor, and HER2. Tumor, Nodes, Metastasis (TNM) staging was performed according to the American Joint Committee on Cancer and the International Union for Cancer Control staging manual, 8th edition (UICC.org). BRCA1 or BRCA2 mutation status was not examined in this study.

This study was approved by the Research Ethics Committee at Jichi Medical University, and written informed consent was obtained from each study participant.

RNA extraction. Total RNA was extracted from the samples using the Illustra RNAspin Mini RNA Isolation kit (GE Healthcare UK) according to the manufacturer's instructions. To assess RNA quality and yield, A260/A280 and A260/A230 ratios for RNA samples were analyzed using a NanoDrop ND-1000 spectrophotometer (NanoDrop Technologies, Inc.). Only RNA with an A260/A280 ratio >1.8 was used for subsequent experiments.

DNA extraction. Dissected tissues or cultured cells were placed in buffered proteinase $\mathrm{K}$ solution at $56^{\circ} \mathrm{C}$ for $3 \mathrm{~h}$, and genomic DNA was isolated and purified using an EZ1 Advanced XL and an EZ1 DNA tissue kit (Qiagen) according to manufacturer's instructions. DNA purity was assessed using a NanoDrop ND-1000 spectrophotometer (NanoDrop Technologies, Inc.) at A260 and A280, with the A260/A280 ratio >1.8 in all instances.

Real-time reverse transcription (RT)-PCR. RT was performed using a High Capacity RNA-to-cDNA kit (Applied Biosystems) with thermal cycling of $37^{\circ} \mathrm{C}$ for $60 \mathrm{~min}$, followed by $95^{\circ} \mathrm{C}$ for 5 min and maintenance at $4^{\circ} \mathrm{C}$. Real-time RT-PCR assays were performed using SYBR Green technology, SYBR Premix Ex Taq reagents (Tli RNaseH Plus; Applied Biosystems) and the QuantStudio 12K Flex real-time PCR system (Applied Biosystems). Thermal cycling conditions were as follows: $95^{\circ} \mathrm{C}$ for $30 \mathrm{sec}$ for denaturation, followed by 40 cycles of $95^{\circ} \mathrm{C}$ for $15 \mathrm{sec}$ and $58^{\circ} \mathrm{C}$ for $1 \mathrm{~min}$ as the cycling stage, $95^{\circ} \mathrm{C}$ for $15 \mathrm{sec}$, $60^{\circ} \mathrm{C}$ for $1 \mathrm{~min}$, and $95^{\circ} \mathrm{C}$ for $15 \mathrm{sec}$ as the melting-curve stage. Gene expression was determined using fluorescence-intensity 
Table I. Clinicopathological features of the breast cancer patients and multivariate analysis.

A, Clinicopathological features of the patients with single breast cancer (SBC), bilateral breast cancer (BBC), and multiple primary cancer (MPC)

\begin{tabular}{|c|c|c|c|c|c|}
\hline Variables & $\operatorname{SBC}(n=120)$ & $\mathrm{BBC}(\mathrm{n}=27)$ & $\mathrm{P}$-value ${ }^{\mathrm{c}}$ & MPC $(n=27)$ & P-value ${ }^{d}$ \\
\hline $\operatorname{Age}^{\mathrm{a}}$ (years) $(<50 / \geq 50)$ & $33 / 87$ & $3 / 24$ & 0.0861 & & \\
\hline $\operatorname{Age}^{\mathrm{b}}$ (years) $(<63 / \geq 63)$ & $61 / 59$ & & & $11 / 16$ & 0.398 \\
\hline BMI $\left(\mathrm{kg} / \mathrm{m}^{2}\right)(\geq 25 /<25)$ & $37 / 83$ & $9 / 18$ & 0.821 & $6 / 21$ & 0.485 \\
\hline Family history (negative/positive) & $104 / 16$ & $23 / 4$ & 0.764 & $25 / 2$ & 0.528 \\
\hline HER 2 ( (negative/positive) & $69 / 37$ & $16 / 9$ & 1.00 & $10 / 10$ & 0.216 \\
\hline $\mathrm{ER}^{\mathrm{e}}$ (negative/positive) & $29 / 77$ & $5 / 20$ & 0.613 & $6 / 14$ & 0.791 \\
\hline TNBC $^{\mathrm{e}}$ (negative/positive) & $88 / 18$ & $21 / 4$ & 1.00 & $18 / 2$ & 0.738 \\
\hline $\mathrm{T}^{\mathrm{e}}(0 / 1 / 2 / 3 / 4)$ & $18 / 55 / 38 / 6 / 9$ & $10 / 15 / 6 / 1 / 0$ & 0.113 & $7 / 16 / 2 / 0 / 0$ & 0.0255 \\
\hline $\mathrm{N}^{\mathrm{e}}(0 / 1,2)$ & $87 / 39$ & $25 / 9$ & 0.678 & $21 / 5$ & 0.342 \\
\hline $\mathrm{M}(0 / 1)$ & $113 / 6$ & $25 / 2$ & 0.641 & $27 / 0$ & 0.593 \\
\hline $1 y^{\mathrm{e}}(0 / 1 / 2 / 3)$ & $35 / 54 / 2 / 2$ & $8 / 10 / 0 / 1$ & 0.679 & $9 / 7 / 0 / 1$ & 0.352 \\
\hline $\mathrm{v}^{\mathrm{e}}(0 / 1 / 2)$ & $49 / 42 / 2$ & $11 / 8 / 0$ & 0.865 & $11 / 6 / 0$ & 0.601 \\
\hline rSAT of tumor tissue [median (range)] & $1.20(-0.0139-3.44)$ & $1.89(0.201-3.28)$ & 0.0312 & $1.79(0.690-2.60)$ & 0.0420 \\
\hline rSAT of normal tissue [median (range)] & $1.88(-0.0331-3.38)$ & $2.50(1.19-3.87)$ & 0.119 & $2.32(1.03-3.87)$ & 0.407 \\
\hline
\end{tabular}

B, Multivariate analysis to predict MPC

\begin{tabular}{|c|c|c|c|c|c|c|}
\hline \multirow[b]{2}{*}{ Variables } & \multicolumn{3}{|c|}{ Multivariate analysis } & \multicolumn{3}{|c|}{ Stepwise multivariate analysis } \\
\hline & OR & $95 \% \mathrm{CI}$ & P-value & OR & $95 \%$ CI & P-value \\
\hline $\mathrm{T}^{\mathrm{f}}(0,1$ vs. $2,3,4)$ & 8.07 & $1.79-36.3$ & 0.00652 & 8.07 & $1.79-36.3$ & 0.00652 \\
\hline rSAT of tumor tissue ${ }^{\mathrm{g}}(>1.5$ vs. $\leq 1.5)$ & 2.96 & $1.15-7.61$ & 0.0243 & 2.96 & $1.15-7.61$ & 0.0243 \\
\hline
\end{tabular}

${ }^{\mathrm{a} A g e}$ cut-off value 50 was determined as referring to a report which indicated that patients aged $<50$ years harbored a higher risk of contralateral breast cancer. ${ }^{b}$ Age cut-off value of 63 years was determined by the median value. ${ }^{\mathrm{C}} \mathrm{P}$-value was calculated by Fisher's exact test or one-way ANOVA followed by Dunnett's 'many-to one' post hoc test between variables of SBC and BBC. Dunnett's 'many-to one' post hoc test showed significant difference between SBC and BBC in tumor tissues. ${ }^{\mathrm{P}} \mathrm{P}$-value was calculated by Fisher's exact test or one-way ANOVA followed by Dunnett's 'many-to one' post hoc test between variables of SBC and MPC. Dunnett's 'many-to one' post hoc test showed significant difference between SBC and MPC in tumor tissues. ${ }^{e}$ These variables could be counted more than twice because of the number of tissue. ${ }^{\mathrm{f}}$ The cut-off value of 1 was determined by the median value. ${ }^{g} \mathrm{ROC}$ determined the cut-off value of 1.5 for multivariate analysis. BMI, body mass index; HER2, human epidermal growth factor receptor 2; ER, estrogen receptor; TNBC, triple-negative breast cancer; T, tumor size; N, lymph node metastasis; M, distant metastasis; ly, lymphatic invasion; v, venous invasion; rSAT, relative expression of satellite alpha transcripts; OR, odds ratio; $\mathrm{CI}$, confidence interval.

measurements obtained using QuantStudio 12K Flex data analysis (Applied Biosystems). A glyceraldehyde 3-phosphate dehydrogenase $(G A P D H)$ fragment was amplified as an internal control. Primers targeting satellite alpha (forward, AAGGTCAATGGCAGAAAAGAA and reverse, CAACGA AGGCCACAAGATGTC) and GAPDH (forward, GAAGGT GAAGGTCGGAGT and reverse, GAAGATGGTGATGGG ATTTC) were used. Log (SAT/GAPDH expression) values were calculated from the mean measurements and represented relative to SAT (rSAT) levels.

Statistical analysis. Fisher's exact test comparing single breast cancer (SBC) to bilateral breast cancer (BBC) used a cut-off value for patient age at 50 years based on a report indicating that patients aged $<50$ years have a higher cumulative 10 -year risk of contralateral breast cancer, despite a lack of
$B R C A$ mutations (27). Fisher's exact test comparing SBC to MPC used a cut-off value for patient age as the median value (i.e., 63 years). Other cut-off values were determined by median values. Cut-off values for the relative SAT-expression levels were determined by receiver operating characteristic (ROC) curve analysis. All statistical analyses were performed using EZR (v.2.4; Saitama Medical Center, Jichi Medical University, Saitama, Japan), which is a graphical user interface for $\mathrm{R}$ (v.3.4.1; The R Foundation for Statistical Computing, Vienna, Austria) (28). Fisher's exact test was used to examine associations between two categorical variables. Continuous variables such as relative SAT-expression (rSAT) levels were evaluated in Kolmogorov-Sminov's test and Bartlett's test, which showed they were normally distributed and homoscedastic. Thereafter, rSAT levels were compared with one-way ANOVA followed by Dunnett's 'many-to one' post hoc test. Medians (ranges) of 
A

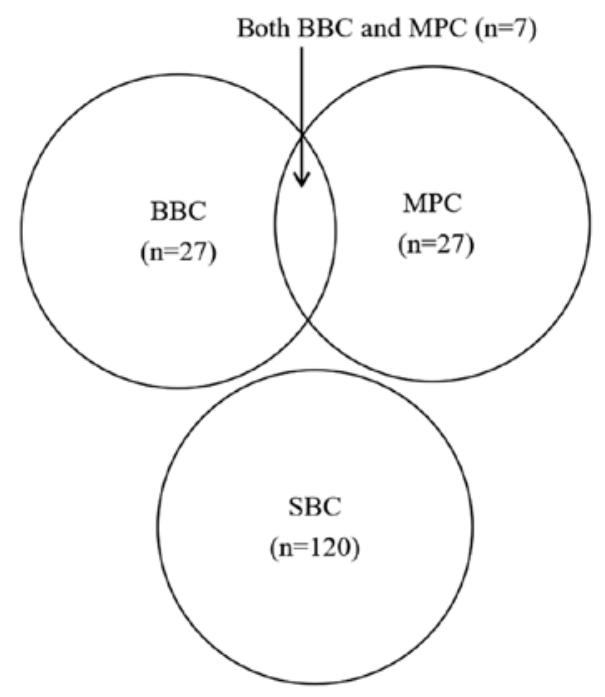

B

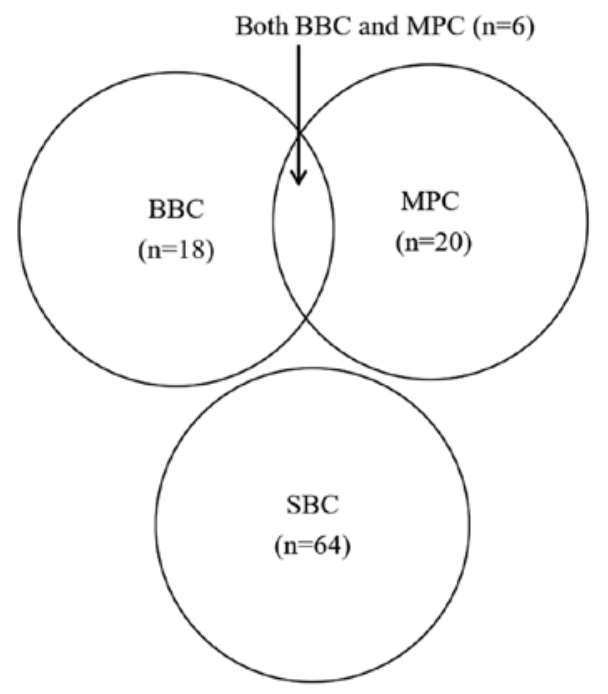

Figure 1. Three groups of patients with SBC, BBC, and MPC. (A) Patients were divided into three groups according to patient clinical characteristics: SBC $(n=120), B B C(n=27)$ and MPC $(n=27)$. Seven patients were included in both the BBC and MPC groups. Statistical analyses were used to compare SBC to $\mathrm{BBC}$ and SBC to MPC. (B) Patients harboring no BRCA-related clinical features were divided into three groups according to their clinical characteristics: SBC $(n=64)$, BBC $(n=18)$ and MPC $(n=20)$. Six patients were included in both BBC and MPC. BBC, bilateral breast cancer; SBC, single breast cancer; MPC, multiple primary cancer.

A

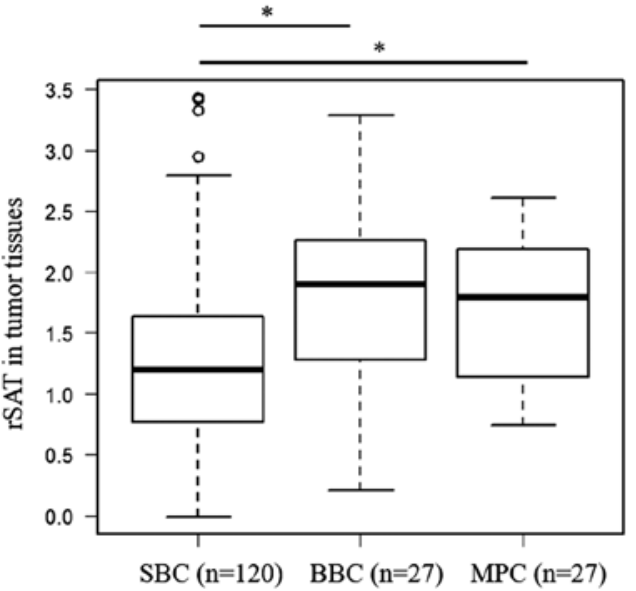

B

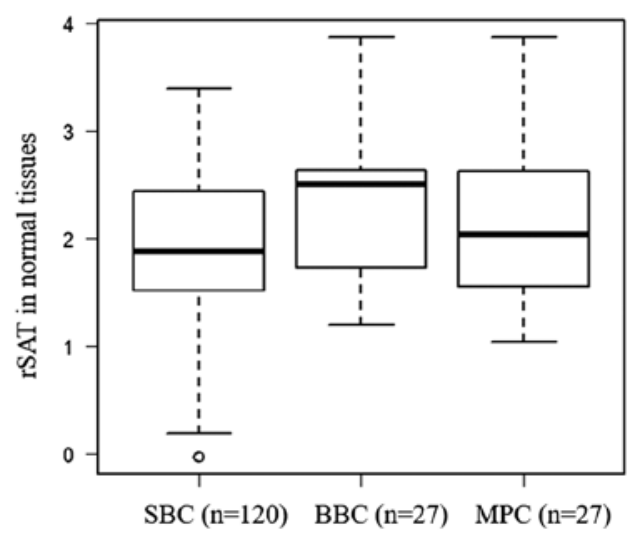

Figure 2. Comparison of rSAT levels between patients with SBC, BBC and MPC. Significant difference in rSAT levels was determined in the tumor tissues, (A) but not in the normal tissues. (B) The y-axis indicates rSAT levels calculated as log (SAT/GAPDH) as determined by RT-qPCR. The error bar represents the standard deviation. ${ }^{*} \mathrm{P}<0.05$. rSAT, relative expression of satellite alpha transcripts; BBC, bilateral breast cancer; SBC, single breast cancer; MPC, multiple primary cancer.

continuous variables are presented in each table. Multivariate analysis was performed by logistic regression. The level of statistical significance was set at $\mathrm{P}<0.05$ unless otherwise specified.

\section{Results}

Comparisons of clinical and pathological features in patients with breast cancer. Our analysis included 167 patients divided into three groups according to their clinical characteristics (Fig. 1A) including 120 patients with SBC (which excluded those with MPC), 27 patients with BBC (including 13 with synchronous BBC and 14 with metachronous BBC), and
27 patients with MPC (including 4 with cervical cancer, 4 with gastric cancer, 3 with colorectal cancer, 3 with pancreatic cancer or IPMN, 2 with uterine cancer, 2 with ovarian cancer, 2 with myelodysplastic syndromes and lymphoma, and one each with lung cancer, thyroid cancer, biliary tract cancer, tongue cancer, renal cancer, and retroperitoneal sarcoma). Seven patients were included in both the BBC and MPC groups. Clinicopathological factors of SBC patients were compared to those of the BBC and MPC cases (Table I).

Relative expression level of SAT (rSAT) in patients with SBC, $B B C$ or $M P C$. We measured rSAT levels in tumor tissues (Fig. 2A) and normal tissues (Fig. 2B) in each group. First, 
Table II. Clinicopathological features of the 96 patients who harbored no BRCA-related clinical features.

\begin{tabular}{|c|c|c|}
\hline \multirow[b]{2}{*}{ Variables } & \multicolumn{2}{|c|}{ Data values } \\
\hline & (median or $\mathrm{n}$ ) & (range or \%) \\
\hline Age (years) & 66 & $50-89$ \\
\hline $\mathrm{BMI}\left(\mathrm{kg} / \mathrm{m}^{2}\right)$ & 23 & $14-38$ \\
\hline Follow-up period (months) & 25.5 & $1.16-38.7$ \\
\hline BBC (negative/positive) & $78 / 18$ & $81.2 / 18.7$ \\
\hline HER2a (negative/positive) & 46/37 (NA20) & $44.7 / 35.9$ \\
\hline $\mathrm{ER}^{\mathrm{a}}$ (negative/positive) & 10/73 (NA20) & $9.71 / 70.9$ \\
\hline MPC (negative/positive) & $76 / 20$ & $79.2 / 20.8$ \\
\hline $\mathrm{T}^{\mathrm{a}}(0 / 1 / 2 / 3 / 4)$ & 19/49/23/6/4 (NA2) & $18.5 / 47.6 / 22.3 / 5.83 / 3.88$ \\
\hline $\mathrm{N}^{\mathrm{a}}(0 / 1,2)$ & $76 / 27$ & $73.8 / 26.2$ \\
\hline $\mathrm{M}(0 / 1)$ & $92 / 4$ & $95.8 / 4.16$ \\
\hline $1 y^{a}(0 / 1 / 2 / 3)$ & 28/40/1/3 (NA31) & $27.2 / 38.8 / 0.97 / 2.91$ \\
\hline$v^{a}(0 / 1 / 2)$ & 39/32/1 (NA31) & $37.9 / 31.1 / 0.97$ \\
\hline
\end{tabular}

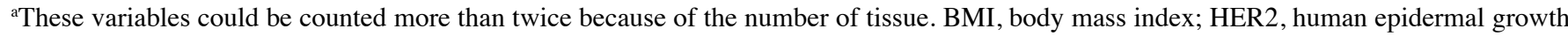
factor receptor 2; ER, estrogen receptor; TNBC, triple-negative breast cancer; T, tumor size; N, lymphnode metastasis; M, distant metastasis; ly, lymphatic invasion; v, venous invasion; rSAT, relative expression of satellite alpha transcripts; NA, not available.

we performed univariate analysis to determine the association between several factors and the probability of developing BBC. Comparing SBC with BBC, univariate analysis revealed that the rSAT level in tumor tissues was the only significant factor associated with BBC development [1.20 (-0.0139-3.44) in SBC vs. $1.89(0.201-3.28)$ in $\mathrm{BBC}$; $\mathrm{P}=0.0312]$ (Table IA and Fig. 2A). Comparing SBC with MPC, univariate analysis revealed that $\mathrm{T}(\mathrm{P}=0.0255)$ and $\mathrm{rSAT}$ level in tumor tissues [1.20 (-0.0139-3.44) in SBC vs. 1.79 (0.690-2.60) in MPC; $\mathrm{P}=0.0420]$ were significantly associated with MPC development (Table IA and Fig. 2A).

For multivariate analysis, we established cut-off values for rSAT level (according to ROC curve analysis) of 1.58 [area under the ROC curve (AUC): $0.649,95 \%$ confidence interval (CI): 0.534-0.764; sensitivity, 0.735; specificity, 0.621) for tumor tissues in BBC and 1.54 (AUC: 0.679, 95\% CI: 0.578-0.780; sensitivity, 0.709; specificity, 0.615) for tumor tissues in MPC. Based on these data, we utilized a cut-off value of 1.5 for multivariate analysis. Similarly, in normal tissues, the cut-off values were 2.47 (AUC: 0.615, 95\% CI: 0.481-0.750; sensitivity, 0.770; specificity, 0.542) for rSAT level in BBC and 2.32 (AUC: 0.559, 95\% CI: 0.406-0.713; sensitivity, 0.689; specificity, 0.421) for rSAT level in MPC. Based on these data, we utilized a cut-off value of 2.4 for multivariate analysis. Multivariate stepwise logistic regression analysis showed that high rSAT levels in tumor tissue (cut-off: 1.5 , determined by ROC) and low $\mathrm{T}$ ( $\mathrm{T}=0.1$; cut-off: 1 , determined by the median) were retained as significant factors [odds ratio (OR): $2.96,95 \%$ CI: $1.15-7.61$; $\mathrm{P}=0.0243$ for rSAT level in tumor tissues; and OR: 8.07, 95\% CI: 1.79-36.3; $\mathrm{P}=0.00652$ for $\mathrm{T}$ ) (Table IB).

However, we failed to confirm the significance of high rSAT levels in normal tissues. We excluded 71 patients with $B R C A$-related clinical features, including a family history of breast cancer, pathological TNBC (including 18 patients with SBC, 4 with $\mathrm{BBC}$, and 2 with MPC), history of ovarian cancer, and younger age ( $<50$ years). We then performed analyses using the remaining 96 patients (Table II), who were divided into three groups [i.e., SBC $(n=64)$, BBC ( $=18$; including 10 patients with synchronous $\mathrm{BBC}$ and 8 with metachronous $\mathrm{BBC}$ ), and MPC $(n=20$; including 3 with cervical cancer, 4 with gastric cancer, 2 with colorectal cancer, 3 with pancreatic cancer or IPMN, 1 with uterine cancer, 2 with myelodysplastic syndromes and lymphoma, and 1 each with lung cancer, thyroid cancer, biliary tract cancer, renal cancer, and retroperitoneal sarcoma)] (Table IIIA and Fig. 1B). Univariate analysis revealed that $\mathrm{T}(\mathrm{P}=0.0328)$, rSAT level in tumor tissues $[1.08(-0.0139-2.79)$ in SBC vs. $1.95(0.736-3.28)$ in $\mathrm{BBC} ; \mathrm{P}=0.000330$ ] (Fig. 3A), and rSAT level in normal tissues [1.74 (-0.0331-3.38) in SBC vs. 2.53 (1.38-3.87) in $\mathrm{BBC} ; \mathrm{P}=0.0310$ ] (Fig. 3B) were significant factors for $\mathrm{BBC}$ development (Table IIIA). Multivariate stepwise logistic regression analysis showed that high rSAT level in normal tissues (cut-off: 2.4, determined by ROC) was the only statistically significant factor (OR: 22.7, 95\% CI: 3.43-151; $\mathrm{P}=0.00120$ ) (Table IIIB). Comparing SBC and MPC, univariate analysis revealed that $\mathrm{T}(\mathrm{P}=0.00960)$ and $\mathrm{rSAT}$ level in tumor tissues [1.08 (-0.0139-2.79) in SBC vs. 1.71 (0.693-2.50) in MPC; $\mathrm{P}=0.0286]$ (Fig. 3A) and rSAT level in normal tissues [1.74 (-0.0331-3.38) in SBC vs. 2.51 (1.41-3.87) in $\mathrm{MPC} ; \mathrm{P}=0.0267$ ] (Fig. 3B) were significant factors for MPC development (Table IIIA). Additionally, multivariate stepwise logistic regression analysis showed that high rSAT level in normal tissues (cut-off: 2.4 , determined by ROC) was the only statistically significant factor (OR: 13.0, 95\% CI: 2.09-81.0; $\mathrm{P}=0.00601$ ) (Table IIIC). In total, 20.0\% of normal tissues showed high rSAT levels ( $>2.4$ ) among all 167 patients, and $15.5 \%$ of normal tissues showed high rSAT levels (>2.4) among 96 patients harboring no $B R C A$-related features (Table II). These data suggested that 15 to $20 \%$ of 
Table III. Clinicopathological features of patients who harbor no $B R C A$-related clinical features and multivariate analyses to predict BBC and MPC.

A, Clinicopathological features of patients who harbored no BRCA-related clinical features with single breast cancer (SBC), bilateral breast cancer (BBC) and multiple primary cancer (MPC)

\begin{tabular}{|c|c|c|c|c|c|}
\hline Variables & $\operatorname{SBC}(n=64)$ & $\operatorname{BBC}(n=18)$ & P-value ${ }^{b}$ & MPC $(n=20)$ & P-value \\
\hline $\operatorname{Age}^{\text {a }}$ (years) $(<63 / \geq 63)$ & $22 / 41$ & $11 / 7$ & 0.0593 & $9 / 14$ & 0.801 \\
\hline BMI $\left(\mathrm{kg} / \mathrm{m}^{2}\right)(\geq 25 /<25)$ & $26 / 38$ & $9 / 8$ & 0.783 & $7 / 13$ & 0.795 \\
\hline HER $2^{\mathrm{d}}$ (negative/positive) & $31 / 25$ & $9 / 7$ & 1.00 & $7 / 8$ & 0.574 \\
\hline $\mathrm{ER}^{\mathrm{d}}$ (negative/positive) & $8 / 48$ & $1 / 15$ & 0.673 & $2 / 13$ & 1.00 \\
\hline $\mathrm{T}^{\mathrm{d}}(0 / 1 / 2 / 3 / 4)$ & $6 / 31 / 18 / 6 / 4$ & $8 / 9 / 4 / 0 / 0$ & 0.0328 & $7 / 12 / 1 / 0 / 0$ & 0.00960 \\
\hline $\mathrm{N}^{\mathrm{d}}(0 / 1,2)$ & $44 / 21$ & $19 / 4$ & 0.281 & $4 / 19$ & 0.284 \\
\hline $\mathrm{M}(0 / 1)$ & $61 / 2$ & $17 / 1$ & 0.535 & $19 / 1$ & 0.547 \\
\hline $1 y^{d}(0 / 1 / 2 / 3)$ & $18 / 31 / 1 / 2$ & $5 / 4 / 0 / 1$ & 0.438 & $7 / 6 / 0 / 0$ & 0.668 \\
\hline$v^{d}(0 / 1 / 2)$ & $27 / 24 / 1$ & $6 / 4 / 0$ & 0.783 & $9 / 4 / 0$ & 0.487 \\
\hline rSAT of tumor tissue [median (range)] & $1.08(-0.0139-2.79)$ & $1.95(0.736-3.28)$ & 0.000330 & $1.71(0.693-2.50)$ & 0.0286 \\
\hline rSAT of normal tissue [median (range)] & $1.74(-0.0331-3.38)$ & $2.53(1.38-3.87)$ & 0.0310 & $2.51(1.41-3.87)$ & 0.0267 \\
\hline
\end{tabular}

$\mathrm{B}$, Multivariate analysis to predict $\mathrm{BBC}$ in patients who do not have $B R C A$-related clinical features

\begin{tabular}{|c|c|c|c|c|c|c|}
\hline \multirow[b]{2}{*}{ Variables } & \multicolumn{3}{|c|}{ Multivariate analysis } & \multicolumn{3}{|c|}{ Stepwise multivariate analysis } \\
\hline & OR & $95 \%$ CI & P-value & OR & $95 \% \mathrm{CI}$ & P-value \\
\hline $\mathrm{T}^{\mathrm{e}}(0,1$ vs. $2,3,4)$ & 2.51 & $0.349-18.0$ & 0.361 & Excluded & & \\
\hline rSAT of tumor tissue ${ }^{\mathrm{f}}(>1.5$ vs. $\leq 1.5)$ & 7.11 & $0.664-76.0$ & 0.105 & Excluded & & \\
\hline rSAT of normal tissue ${ }^{\mathrm{g}}(>2.4$ vs. $\leq 2.4$ ) & 14.2 & $1.74-117$ & 0.0133 & 22.7 & $3.43-151$ & 0.00120 \\
\hline
\end{tabular}

C, Multivariate analysis to predict MPC in patients who do not have $B R C A$-related clinical features

\begin{tabular}{|c|c|c|c|c|c|c|}
\hline \multirow[b]{2}{*}{ Variables } & \multicolumn{3}{|c|}{ Multivariate analysis } & \multicolumn{3}{|c|}{ Stepwise multivariate analysis } \\
\hline & OR & $95 \% \mathrm{CI}$ & P-value & OR & $95 \% \mathrm{CI}$ & P-value \\
\hline $\mathrm{T}^{\mathrm{h}}(0,1$ vs. $2,3,4)$ & - & 0.00 -inf & 0.994 & Excluded & & \\
\hline rSAT of tumor tissue $(>1.5$ vs. $\leq 1.5$ ) & 1.20 & $0.164-8.80$ & 0.858 & Excluded & & \\
\hline rSAT of normal tissue ${ }^{\mathrm{j}}(>2.4$ vs. $\leq 2.4$ ) & 10.0 & $0.777-129$ & 0.0774 & 13.0 & $2.09-81.0$ & 0.00601 \\
\hline
\end{tabular}

${ }^{a}$ The age cut-off value of 63 years was determined by the median value. ${ }^{\text {} P} \mathrm{P}$-value was calculated by Fisher's exact test or one-way ANOVA followed by Dunnett's 'many-to one' post hoc test between variables of SBC and BBC. Dunnett's 'many-to one' post hoc test showed significant difference between SBC and BBC in both tumor and normal tissues. 'P-value was calculated by Fisher's exact test or one-way ANOVA followed by Dunnett's 'many-to one' post hoc test between variables of SBC and MPC. Dunnett's 'many-to one' post hoc test showed significant difference between SBC and MPC in both tumor and normal tissues. 'These variables could be counted more than twice because of the

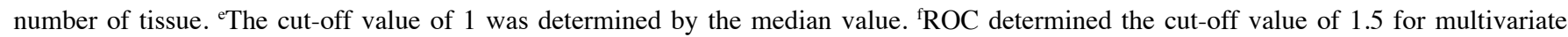
analysis. ${ }^{g}$ ROC determined the cut-off value of 2.4 for multivariate analysis. ${ }^{h}$ The cut-off value of 1 was determined by the median value. ${ }^{i}$ ROC determined cut-off value of 1.5 for multivariate analysis. ${ }^{j}$ ROC determined cut off value of 2.4 for multivariate analysis. BMI, body mass index; HER2, human epidermal growth factor receptor 2; ER, estrogen receptor; TNBC, triple-negative breast cancer; T, tumor size; N, lymphnode metastasis; M, distant metastasis; rSAT, relative expression of satellite alpha transcripts; OR, odds ratio; CI, confidence interval.

patients had a high risk of developing BBC and MPC based on normal tissue features.

\section{Discussion}

We demonstrated that satellite alpha transcripts (SAT) in tumor tissues were involved in the development of BBC and MPC in 167 patients with breast cancer. However, in our initial analysis, SAT in normal tissues did not have predictive value, which might be explained by selection bias of the SBC patients harboring $B R C A$-related clinical features and higher or lower rSAT levels in normal tissues. We excluded these patients for sub-analysis, finding higher normal-tissue rSAT levels in patients with both BBC 
A

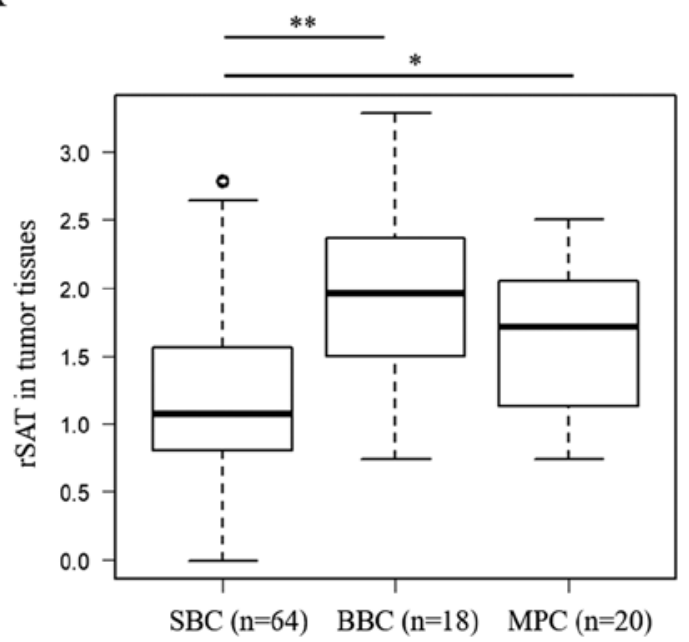

B

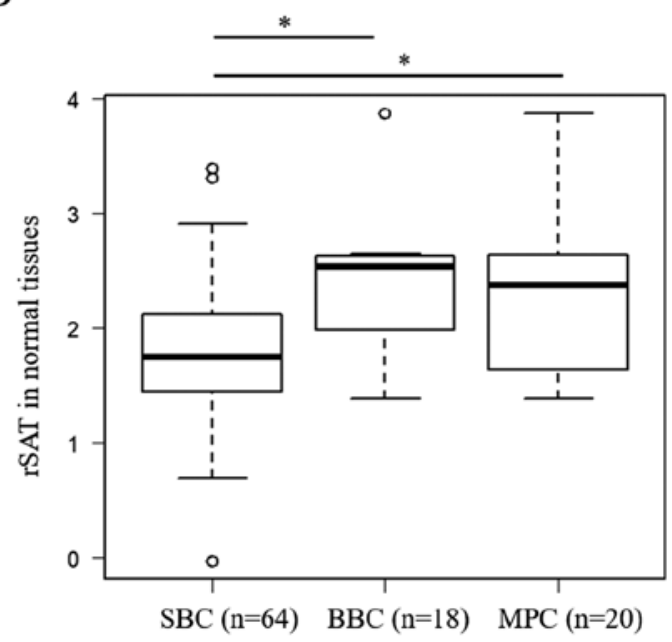

Figure 3. Comparison of rSAT levels between SBC, BBC and MPC from patients lacking BRCA-related clinical features. Significant difference in rSAT levels was determined in the tumor tissues (A) and in the normal tissues. (B) The y-axis indicates rSAT levels calculated as log (SAT/GAPDH) as determined by RT-qPCR. The error bar represents the standard deviation. ${ }^{*} \mathrm{P}<0.05,{ }^{* *} \mathrm{P}<0.001$. rSAT, relative expression of satellite alpha transcripts; BBC, bilateral breast cancer; SBC, single breast cancer; MPC, multiple primary cancer.

and MPC relative to those in patients with SBC. In these selected patients with breast cancer, multivariate analysis revealed rSAT levels $>2.4$ in normal tissues as a significant predictor of development of $\mathrm{BBC}(\mathrm{OR}, 22.7 ; \mathrm{P}=0.00120)$ and MPC (OR, 13.0; $\mathrm{P}=0.00601)$. Our data indicated that patients with breast cancer and exhibiting high rSAT levels in normal breast tissues had a 10 - to 20 -fold increased risk for the development of multiple cancers when they harbored no BRCA-related clinical features. These patients had been excluded from the high-risk group for BBC; however, our subsequent results highlighted unique findings.

Well-established risk factors for bilateral breast cancer include young age, a family history of breast cancer, and TNBC, which are strongly associated with familial cancer due to a $B R C A$ mutation $(1-5,27)$. Nevertheless, some noncarriers of $B R C A$ mutations show a high cumulative risk for $\mathrm{BBC}$ and similar to that in women with $B R C A$ mutations (27). The prevalence of $B R C A$ mutations was found to be $16.3 \%$ (34/209) for Korean breast cancer patients with BBC, implying that genetic testing for $B R C A$ is insufficient to determine predisposition for BBC (29). Moreover, despite the increased risk for developing $\mathrm{BBC}$ in patients harboring these factors, few clinical risk factors for BBC have been identified.

Epigenetic alterations are frequently observed in normal tissues surrounding cancer tissues and can contribute to epigenetic field cancerization. Epigenetic alterations have been identified in many types of human cancers in connection with carcinogenesis in the absence of genetic sequence abnormalities $(22-24,30)$ and associated with chromosomal instability. We previously evaluated DNA demethylation and its targets, including repetitive sequences, LINE-1, and SAT, demonstrating that increased SAT-demethylation levels in normal tissues of the stomach are associated with susceptibility to multiple gastric cancers (21), and that LINE-1 demethylation levels in normal tissues of patients with colon cancer are associated with multiple primary colon cancer $(10,21)$. These results indicate that demethylation of satellite DNAs plays an important role in field cancerization, resulting in the development of multiple primary cancers. Moreover, our previous study revealed that rSAT levels were correlated significantly with levels of SAT hypomethylation (25), indicating that the rSAT level is involved in the development of multiple cancers. Additionally, a study of mitotic errors in SAT-transfected cells indicated that SAT overexpression induces chromosomal alterations (25). Our data combined with those of previous studies indicate a potential role of epigenetic alterations in field cancerization and its contribution to the development of multiple cancers of the stomach, colon and breast. The possibility that demethylation-associated overexpression of satellite sequences and its correlation with chromosomal instability results in field cancerization underlying the development of multiple cancers is highly speculative. Furthermore, it is possible that field cancerization may occur prior to the presence of chromosomal instability.

Regarding prophylactic surgery, of 496,488 women diagnosed with unilateral invasive breast cancer, $59.6 \%$ underwent breast-conserving surgery, 33.4\% underwent unilateral mastectomy, and $7.0 \%$ underwent CPM; however, the survival benefits remain controversial. Mortality does not differ between bilateral mastectomy and breast-conserving surgery plus radiation (16), and studies report that prophylactic mastectomy can decrease the risk of future breast cancer by 90 to $97 \%$ (31). The National Comprehensive Cancer Network Guidelines and Preventive Service Task Force Recommendations suggest that prophylactic mastectomy should be part of the discussion among patients who test positive for $B R C A$ mutations or have a strong family history of breast cancer (32). Howard-McNattesal reported that $37 \%$ of $B R C A$-negative patients at their institution chose contralateral prophylactic mastectomy, and another study reported that $83.4 \%$ of patients who underwent contralateral prophylactic mastectomy did not have a known BRCA mutation (33). Contralateral prophylactic mastectomy is acceptable to reduce fear or to circumvent the risk of developing a second primary 
breast cancer, but insufficient surgery should be avoided. In our study, patients with rSAT levels $>2.4$ in normal tissues had a 20-fold increased risk of $\mathrm{BBC}$ when lacking $B R C A$-related clinical features. Our data provide important information for treatment decisions.

MPC in other organs is also likely to develop in patients with breast cancer. Massive autopsy data demonstrate that patients with breast cancer have a generally increased risk of developing MPC in other organs, such as ovarian, pancreatic, and skin cancer, and a decreased incidence of colorectal and cervical cancer (6). Patients in our study developed six gynecologic (22.2\% in patients with MPC), seven gastrointestinal $(25.9 \%$ in patients with MPC), and three pancreatic cancers (11.1\% in patients with MPC), as well as other types of cancer. Kato et al (34) reported that $7.58 \%$ of patients develop primary breast cancer after the resection of colorectal cancer. In the present study, our data revealed a high frequency of gynecologic and gastrointestinal MPCs, suggesting the need for wide surveillance, from gynecologic to gastrointestinal areas, for patients who harbor higher rSAT levels in normal tissues.

This study has limitations. The normal breast tissue was donated during surgical operations, and SAT-expression levels in normal tissues could only be assessed after surgery. It is difficult to accurately obtain normal breast tissues around malignant tumors by preoperative biopsy; however, it is possible to resolve the difficulty of obtaining normal tissue samples preoperatively by measuring SAT preoperatively using plasma samples. Kondratova et al (35) measured two subtypes of satellite DNA (HSATII and GSATII) in blood plasma by RT-PCR, finding that transcript levels differed between healthy donors and patients with colon cancer. Similarly, plasma SAT levels in patients with breast cancer may be higher than those in healthy donors, and these levels in patients with BBC or MPC may also be higher than those in patients with $\mathrm{SBC}$. These levels might provide a basis for detecting the risk of developing BBC and MPC preoperatively. Moreover, the continual measurement of plasma SAT levels during follow-up might provide insight into the need for surveillance testing.

In conclusion, we demonstrated for the first time a strong association between SAT-expression levels in normal breast tissues and the development of BBC, as well as MPC, in other organs when patients with breast cancer lack $B R C A$-related features. Additionally, these patients exhibited a 22- and 13-fold increased risk for the development of BBC and MPC, respectively. Importantly, these patients lacking $B R C A$-related features had previously been excluded from high-risk BBC categorization; therefore, our study improves the understanding of the risk for the development of BBC and MPC and the need for prophylactic surgery.

\section{Acknowledgements}

Not applicable.

\section{Funding}

This study was supported by a grant-in-aid for Scientific Research (no. JP 17K10562) from the Ministry of Education,
Culture, Sports, Science and Technology and the JKA Foundation through its promotion funds from the Keirin Race [no. 27-1-068 (2)].

\section{Availability of data and materials}

The datasets generated during this study are available from the corresponding author upon reasonable request.

\section{Authors' contributions}

NK designed this study and wrote the initial draft of the manuscript. KS contributed to the analysis and interpretation of data and assisted with the preparation of the manuscript. IA, YE, ST, HI, FW, KI, MS, KF, FK and TR conducted the data collection, analysis and interpretation of the data and critically reviewed the manuscript. All authors read and approved the final version of the manuscript and agree to be accountable for all aspects of the research in ensuring that the accuracy or integrity of any part of the work are appropriately investigated and resolved.

\section{Ethics approval and consent to participate}

This study was approved by the Research Ethics Committee at Jichi Medical University. Written informed consent was obtained from each study participant.

\section{Patient consent for publication}

Not applicable

\section{Competing interests}

The authors declare that they have no competing interests.

\section{References}

1. Beinart G, Gonzalez-Angulo AM, Broglio K, Mejia J, Ruggeri A, Mininberg E, Hortobagyi GN and Valero V: Clinical course of 771 patients with bilateral breast cancer: Characteristics associated with overall and recurrence-free survival. Clin Breast Cancer 7: 867-874, 2007.

2. Chen Y, Thompson W, Semenciw R and Mao Y: Epidemiology of contralateral breast cancer. Cancer Epidemiol Biomarkers Prev 8: 855-861, 1999.

3. Kheirelseid EA, Jumustafa H, Miller N, Curran C, Sweeney K, Malone C, McLaughlin R, Newell J and Kerin MJ: Bilateral breast cancer: Analysis of incidence, outcome, survival and disease characteristics. Breast Cancer Res Treat 126: 131-140, 2011.

4. Hartmann LC, Schaid DJ, Woods JE, Crotty TP, Myers JL, Arnold PG, Petty PM, Sellers TA, Johnson JL, McDonnell SK, et al: Efficacy of bilateral prophylactic mastectomy in women with a family history of breast cancer. $\mathrm{N}$ Engl J Med 340: 77-84, 1999.

5. Li CI, Malone KE, Porter PL and Daling JR: Epidemiologic and molecular risk factors for contralateral breast cancer among young women. Br J Cancer 89: 513-518, 2003.

6. Shibahara Y, Sugawara Y, Miki Y, Hata S, Takahashi H, Nakamura Y, Suzuki T, Ohuchi N, Tsuji I and Sasano H: Analysis of multiple primary cancer autopsy cases associated with breast cancer: 2002-2010. Pathol Int 66: 695-700, 2016.

7. Momoh AO, Cohen WA, Kidwell KM, Hamill JB, Qi J, Pusic AL, Wilkins EG and Matros E: Tradeoffs associated with contralateral prophylactic mastectomy in women choosing breast reconstruction: Results of a prospective multicenter cohort. Ann Surg 266: 158-164, 2017. 
8. Tuttle TM, Habermann EB, Grund EH, Morris TJ and Virnig BA Increasing use of contralateral prophylactic mastectomy for breast cancer patients: A trend toward more aggressive surgical treatment. J Clin Oncol 25: 5203-5209, 2007.

9. Albornoz CR, Matros E, Lee CN, Hudis CA, Pusic AL, Elkin E, Bach PB, Cordeiro PG and Morrow M: Bilateral mastectomy versus breast-conserving surgery for early-stage breast cancer: The role of breast reconstruction. Plast Reconstr Surg 135: $1518-1526,2015$.

10. Kamiyama H, Suzuki K, Maeda T, Koizumi K, Miyaki Y, Okada S, Kawamura YJ, Samuelsson JK, Alonso S, Konishi F and Perucho M: DNA demethylation in normal colon tissue predicts predisposition to multiple cancers. Oncogene 31 : 5029-5037, 2012

11. Braakhuis BJ, Tabor MP, Kummer JA, Leemans CR and Brakenhoff RH: A genetic explanation of Slaughter's concept of field cancerization: Evidence and clinical implications. Cancer Res 63: 1727-1730, 2003.

12. Suzuki K, Suzuki I, Leodolter A, Alonso S, Horiuchi S, Yamashita K and Perucho M: Global DNA demethylation in gastrointestinal cancer is age dependent and precedes genomic damage. Cancer Cell 9: 199-207, 2006.

13. Deng G, Lu Y, Zlotnikov G, Thor AD and Smith HS: Loss of heterozygosity in normal tissue adjacent to breast carcinomas. Science 274: 2057-2059, 1996

14. Ahlquist T, Lind GE, Costa VL, Meling GI, Vatn M, Hoff GS, Rognum TO, Skotheim RI, Thiis-Evensen E and Lothe RA: Gene methylation profiles of normal mucosa, and benign and malignant colorectal tumors identify early onset markers. Mol Cancer 7: 94, 2008.

15. Belshaw NJ, Pal N, Tapp HS, Dainty JR, Lewis MP, Williams MR, Lund EK and Johnson IT: Patterns of DNA methylation in individual colonic crypts reveal aging and cancer-related field defects in the morphologically normal mucosa. Carcinogenesis 31 : $1158-1163,2010$

16. Konishi K, Shen L, Jelinek J, Watanabe Y, Ahmed S, Kaneko K, Kogo M, Takano T, Imawari M, Hamilton SR and Issa JP: Concordant DNA methylation in synchronous colorectal carcinomas. Cancer Prev Res (Phila) 2: 814-822, 2009.

17. Menigatti M, Truninger K, Gebbers JO, Marbet U, Marra G and Schär P: Normal colorectal mucosa exhibits sex- and segment-specific susceptibility to DNA methylation at the hMLH1 and MGMT promoters. Oncogene 28: 899-909, 2009.

18. Paun BC, Kukuruga D, Jin Z, Mori Y, Cheng Y, Duncan M, Stass SA, Montgomery E, Hutcheon D and Meltzer SJ: Relation between normal rectal methylation, smoking status, and the presence or absence of colorectal adenomas. Cancer 116 : 4495-4501, 2010.

19. Ushijima T: Epigenetic field for cancerization. J Biochem Mol Biol 40: 142-150, 2007

20. Worthley DL, Whitehall VL, Buttenshaw RL, Irahara N, Greco SA, Ramsnes I, Mallitt KA, Le Leu RK, Winter J, $\mathrm{Hu} \mathrm{Y}$, et al: DNA methylation within the normal colorectal mucosa is associated with pathway-specific predisposition to cancer. Oncogene 29: 1653-1662, 2010.

21. Saito M, Suzuki K, Maeda T, Kato T, Kamiyama H, Koizumi K, Miyaki Y, Okada S, Kiyozaki H and Konishi F: The accumulation of DNA demethylation in Sat $\alpha$ in normal gastric tissues with Helicobacter pylori infection renders susceptibility to gastric cancer in some individuals. Oncol Rep 27: 1717-1725, 2012

22. Herrera LA, Prada D, Andonegui MA and Dueñas-González A: The epigenetic origin of aneuploidy. Curr Genomics 9: 43-50, 2008 .
23. Kawano H, Saeki H, Kitao H, Tsuda Y, Otsu H, Ando K, Ito S, Egashira A, Oki E, Morita M, et al: Chromosomal instability associated with global DNA hypomethylation is associated with the initiation and progression of esophageal squamous cell carcinoma. Ann Surg Oncol 21 (Suppl 4): S696-S702, 2014.

24. Rodriguez J, Frigola J, Vendrell E, Risques RA, Fraga MF, Morales C, Moreno V, Esteller M, Capellà G, Ribas M and Peinado MA: Chromosomal instability correlates with genome-wide DNA demethylation in human primary colorectal cancers. Cancer Res 66: 8462-9468, 2006.

25. Ichida K, Suzuki K, Fukui T, Takayama Y, Kakizawa N, Watanabe F, Ishikawa H, Muto Y, Kato T, Saito M, et al: Overexpression of satellite alpha transcripts leads to chromosomal instability via segregation errors at specific chromosomes. Int J Oncol: Mar 16, 2018 (Epub ahead of print). doi: 10.3892/ijo.2018.4321 2018.

26. Wolff AC, Hammond ME, Hicks DG, Dowsett $M$, McShane LM, Allison KH, Allred DC, Bartlett JM, Bilous M, Fitzgibbons $\mathrm{P}$, et al: Recommendations for human epidermal growth factor receptor 2 testing in breast cancer: American Society of Clinical Oncology/College of American Pathologists clinical practice guideline update. J Clin Oncol 31: 3997-4013, 2013.

27. Reiner AS, John EM, Brooks JD, Lynch CF, Bernstein L, Mellemkjaer L, Malone KE, Knight JA, Capanu M, Teraoka SN, et al: Risk of asynchronous contralateral breast cancer in noncarriers of BRCA1 and BRCA2 mutations with a family history of breast cancer: A report from the Women's Environmental Cancer and Radiation Epidemiology Study. J Clin Oncol 31: 433-439, 2013.

28. Kanda Y: Investigation of the freely available easy-to-use software 'EZR' for medical statistics. Bone Marrow Transplant 48: 452-458, 2013.

29. Kang E, Seong MW, Park SK, Lee JW, Lee J, Kim LS, Lee JE, Kim SY, Jeong J, Han SA, et al: The prevalence and spectrum of BRCA1 and BRCA2 mutations in Korean population: Recent update of the Korean Hereditary Breast Cancer (KOHBRA) study. Breast Cancer Res Treat 151: 157-168, 2015.

30. Feinberg AP and Vogelstein B: Hypomethylation distinguishes genes of some human cancers from their normal counterparts. Nature 301: 89-92, 1983.

31. Rebbeck TR, Friebel T, Lynch HT, Neuhausen SL, van't Veer L, Garber JE, Evans GR, Narod SA, Isaacs C, Matloff E, et al: Bilateral prophylactic mastectomy reduces breast cancer risk in BRCA1 and BRCA2 mutation carriers: The PROSE Study Group. J Clin Oncol 22: 1055-1062, 2004.

32. Bevers TB, Armstrong DK, Arun B, Carlson RW, Cowan KH, Daly MB, Fleming I, Garber JE, Gemignani M, Gradishar WJ, et al: Breast cancer risk reduction. J Natl Compr Canc Netw 5: 676-701, 2007.

33. Fu Y, Zhuang Z, Dewing M, Apple S and Chang H: Predictors for contralateral prophylactic mastectomy in breast cancer patients. Int J Clin Exp Pathol 8: 3748-3764, 2015.

34. Kato T, Suzuki K, Muto Y, Sasaki J, Tsujinaka S, Kawamura YJ, Noda H, Horie H, Konishi F and Rikiyama T: Multiple primary malignancies involving primary sporadic colorectal cancer in Japan: Incidence of gastric cancer with colorectal cancer patients may be higher than previously recognized. World J Surg Oncol 13: 23, 2015.

35. Kondratova VN, Botezatu IV,Shelepov VPandLikhtenshtein AV: Transcripts of satellite DNA in blood plasma: Probable markers of tumor growth. Mol Biol (Mosk) 48: 999-1007, 2014 (In Russian). 